Erratum zu: Methoden, Umfrageforschung, Arbeitssoziologie, Soziologie der Mobilität, Religionssoziologie, Extremismusforschung, Mediensoziologie, Literarische Soziologie

\title{
Wolfgang Himmel
}

Online publiziert: 30 . Oktober 2014

(C) Springer Fachmedien Wiesbaden 2014

Erratum zu: Köln Z Soziol (2014) XX

DOI: 10.1007/s11577-014-0256-7

\section{Anmerkung der Redaktion}

In den Literaturbesprechungen von Heft 2 der Kölner Zeitschrift für Soziologie und Sozialpsychologie dieses Jahrgangs wurde irrtümlicherweise aus technischen Gründen die Besprechung von Wolfgang Himmel zu dem Buch Einzelgänger von Wolfgang Sofsky (S. 328-330) unkorrigiert abgedruckt. Da sich die KZfSS darum bemüht, Beiträge den Lesern in möglichst fehlerfreier Semantik und Grammatik der deutschen Sprache zu präsentieren, haben wir uns dazu entschlossen, die Besprechung in korrigierter Fassung erneut abzudrucken.

Sofsky, Wolfgang: Einzelgänger. Matthes \& Seitz, Berlin 2013. 271 Seiten. ISBN: 978-3-88221-032-3. Preis: $€ 19,90$.

\section{Wolfgang Himmel}

„Einzelgänger“ - der Titel dieser Sammlung von Erzählungen des Soziologen Wolfgang Sofsky weckt zunächst Assoziationen an Käuze, Wunderlinge, Ausgestoßene,

Die Onlineversion des Originalbeitrages ist erreichbar unter doi:http://dx.doi.org/10.1007/s11577-014-0256-7

W. Himmel $(\bowtie)$

Institut für Allgemeinmedizin, Georg-August-Universität Göttingen,

Humboldtallee 38, 37073 Göttingen, Deutschland

E-Mail: whimmel@gwdg.de 
mal traurige, mal tragische, mal faszinierende Gestalten. In der Tat, solche Typen gibt es in diesem Buch, zum Beispiel den Eremiten in der Erzählung „Eine Lehrstunde“. Und dort liest man so vertraute, oft schon gehörte Sätze wie: „Aus der Welt sei er geflohen, um Gott zu finden“" (S. 33.). Aber schon dieser klassische Einzelgänger hat etwas Verstörendes. Er kämpft beständig gegen eine Armada von Dämonen, die einem Bild von Hieronymus Bosch entsprungen sein könnten. Ist es diesem Einzelgänger noch gelungen, Frieden mit den Dämonen und mit sich selbst zu finden, erzählen fast alle anderen Gestalten von einem verlorenen oder zumindest unglücklichen Kampf zwischen innerer Ruhe, herbeigesehntem Glück, Leidenschaft und Isolation. Da gibt es eine Statue oder Göttin („Die Unnahbare“), die für alle Betrachter regungslos und kalt, fast möchte man sagen: einer Rolle gleich, auf ihrem Thron sitzt, immer wieder wehrlos der Leidenschaft zur Beute fällt, ohne jedoch Erfüllung zu finden.

Bis zu diesem Zeitpunkt mag man das alles noch allegorisch betrachten, als Zeitdiagnose des modernen, vereinsamten Menschen. Doch langsam zeichnet sich ab, dass Sofsky den Einzelgänger weit grundsätzlicher und als konstitutives Element der Menschheitsgeschichte anlegt. Schon in der ersten Erzählung („Die sechste Stunde“), dem Ursprung der Menschheit, misslingt die Schöpfung: Weder Gott findet im Menschen ein Wesen zur Unterhaltung und Erheiterung, noch der Mensch einen Partner, der das Leben mit ihm teilt. Und „Der Einzelgänger“ - die zweite Erzählung - versucht dann das anthropologische Dilemma sinnbildlich darzustellen: das Gehen, gedacht als Befreiung, Erhebung aus Dreck und Staub, bietet keinen Ausweg, ja, ist ein Irrweg, weil es in permanentem Widerspruch zum Denken steht. Beides gemeinsam ist nicht möglich - und beides vereinzelt den Menschen.

Nun könnte man versuchen, die Erzählungen als variantenreiche Verkörperungen dieser anthropologischen Grundkonstellation zu lesen: Der Einzelgänger wird fast schon zum Wesen der Sozialität - und damit zur Provokation des Grundtopos jeglicher Soziologie. Die Dyade, wie jede Gruppe von Menschen, ist in den Erzählungen immer nur eine Ansammlung von Einzelgängern, die sich beziehungslos, atomistisch bewegen. Da gibt es den Heimkehrer (in „Glockenblumen“), der wie alle anderen Heimkehrer in dieser Gruppe isoliert ist/wird. Zurückgekehrt findet er keine Heimat mehr. Um anzukommen und dazuzugehören, müsste er alles Bisherige vergessen und sicherstellen, dass seine Erscheinung Andere nicht an Vergangenes erinnert. Heimat das ist nur noch das Jetzt der Gesellschaft, ohne Erinnerungen. Alles, was diese Ordnung, was die erinnerungslose Heimat stört, stößt auf Ablehnung und Feindschaft.

Dieser Gedanke kulminiert in einem bizarr erzählten Maskenfest („Das Gericht“"), bei dem der Hofnarr es wagt, zum ersten Mal ohne Maske, denn das soll seine „Maske“ sein, zu erscheinen. In seiner Sichtbarkeit wird er zur unerträglichen Wahrheit, den das Gericht zu einem Spießrutenlaufen verurteilt. Im Moment größter Ausgelassenheit also, beim Karneval, kulminieren Entfremdung und Einsamkeit.

Nicht anders geht es zu bei dem, was wir als Beispiel menschlicher Aktivität schlechthin begreifen, dem Spiel („Ein Handgemenge“). Die Isolation zweier Schachspieler ist so weit fortgeschritten, als ,lebte jeder auf einen anderen Planeten“ (S. 153). Es ist Krieg, den schon das Schachbrett selbst in Sofskys brillanter Beschreibung vorwegnimmt: „Der König saß auf einem Thron, Bischöfe dienten ihm als Läufer und berittene Krieger als Springer. Geistlichkeit und Ritterschaft waren die Säulen der Gesellschaft, im Frieden wie im Krieg. Sie bezahlten ihre Königstreue 
mit Blut und Ideen. Alle hatten sie die Augen weit aufgerissen, aus Überraschung, Angst oder Entsetzen? Die Berserker an den Ecken des Bretts stellten die Türme dar. Voller Kampfeswut entblößten sie ihre Schneidezähne und bissen in den Rand ihrer Schilde“" (S. 149).

Die wenigen Begegnungen in diesem Buch, die gewaltfrei verlaufen, sind fast die kältesten: Die Säuferin und der Säufer, die täglich zusammen in der Kneipe sitzen, zusammen trinken, vereint im Trinken, die die Einsamkeit nicht ertragen, aber kein einziges Wort miteinander wechseln (,Spinnenbeine“). Die gelingende Kommunikation dagegen, fast die einzige in diesem Buch, ist die Kommunikation mit dem eigenen Schatten, meisterhaft beschrieben in der Erzählung „,Gelbes Licht“. Der Protagonistin, die die behüteten Kinder, den selbstsüchtigen Mann, die treulose Freundin als „,vorletzte Lösung“ verlassen hat, bleibt in der Einsamkeit der Hotelzimmer nur der Schatten. Der Einzige, der immer da ist, den man treten kann, der dann zwar zurücktritt, aber keine Schmerzen hinterlässt. Und hier erfahren wir en passant auch, was Krankheit so entsetzlich macht: Auf dem Bett gefesselt, zerdrücken wir unseren Schatten und liegen dann danieder, weil unser Schatten klein und schwach geworden ist.

Es gibt eine andere kommunikative Situation in diesem Buch, die zunächst, trotz der Situation in einem Straflager, ganz entspannt daherkommt. Die Erzählung „Nr. 403“ beginnt als Plauderei zwischen dem Lagerkommandanten und einem Besucher. Fast stellt sich Behaglichkeit bei dieser netten Plauderei ein, bis sich dann der Besucher in einer kafkaesken Wendung ganz am Schluss selbst in die Gruppe der Gefangenen einreihen muss - für ihn war ein Platz die ganze Zeit freigehalten worden.

Es gibt wohl nur eine Geschichte, bei der es nicht zu einer Verletzung kommt - im Gegenteil: Der Cellist in „Tänze“ spielt in einem ekstatischen Konzert eine Sonate von Bach. Nicht nur reißt uns Sofsky hinein in seine sprachlich-geniale Beschreibung der Musik, die ohne weiteres an andere große Umsetzungen von Musik in Sprache erinnert, wie zum Beispiel Hartmut Langes Beschreibung einer Beethoven-Sonate in „Das Konzert“. Bei Sofsky erreicht der Künstler nach einem körperlich erschöpfenden Durchgang durch diese Sonate vollkommenste Erfüllung. Vielleicht deshalb, weil die Figuren auf den Säulen des Gebetsraums, in dem das Konzert stattfand, die einzigen Zuhörer waren. Mit seiner Musik vermag der Cellist, die jahrhundertealten versteinerten Gesichter zur Entzückung zu erwecken.

Man kann sich diesem Buch auf verschiedenen Wegen nähern. Man kann, wie es der Rezensent bisher andeutete, Themen und Motive zu entdecken versuchen, die die Erzählungen miteinander verbinden und gleichzeitig Anknüpfungspunkte - und sei es nur negativer Art - an Wolfgang Sofskys wissenschaftliche Herkunftsdisziplin sind. Man mag vielleicht Sartres Serialität entdecken, Dreitzels Leiden an der Gesellschaft; noch viel mehr gibt es natürlich Bezugspunkte zur Literatur, vorneweg Franz Kafkas absurde Erzählungen. Auch Zweigs Schachnovelle oder Thomas Manns Tod in Venedig scheinen uns zu grüßen. Solche Motive lassen sich unschwer erkennen, aber tragen letztlich nichts zum Verständnis, vor allem aber nichts zum Leseerlebnis bei. Und genau das Leseerlebnis macht die Wucht des Buches aus. Es sind keine dürren Erzählungen, die einer soziologischen These zur Illustration verhelfen sollen. Die Erzählungen folgen einem ganz eigenen Rhythmus, unterstrichen durch eine diszipli- 
nierte Satzstruktur und eine manchmal überbordende Fantasie in der Beschreibung der Umstände.

Der Autor als Literat vermag uns in seine Erzählungen hineinzuziehen - und immer wieder zu überraschen, durch Ideen, Kreativität, und sprachliche Kühnheit. Der Autor als Soziologe vermittelt uns fast brutal die gesellschaftliche Eingebundenheit des Menschen als zunehmende Isolation. Sprachlich treibt er manchmal die Isolation soweit auf die Spitze, dass er die Differenz zwischen zwei Personen gewissermaßen verschleift und das Personalpronomen nicht mehr ganz eindeutig ist, als wäre es egal, um wen es geht. Ehepaare, Liebespaare, Erotik fehlen gänzlich. Vielleicht weiß der Autor, dass ihm der prall-lebendige Stil eines anderen Soziologen und großen Literaten, Elias Canetti, abgeht. Oder will uns der Autor noch eine letzte Hoffnung lassen, die er nicht mit Zynismus, bitterer Sprache und eisiger Kälte seziert? Große Literatur? Große Soziologie? - ein großartiges Buch! 